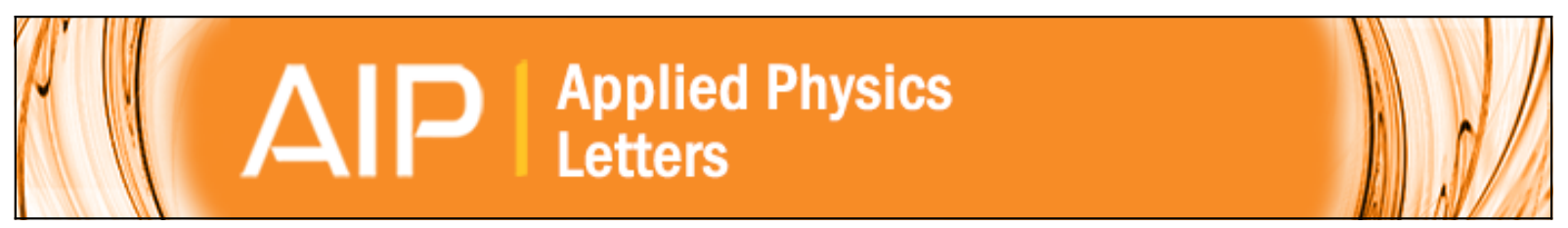

\title{
Anomalous sound absorption in lattices of cylindrical perforated shells
}

Victor M. García-Chocano and José Sánchez-Dehesa

Citation: Applied Physics Letters 106, 124104 (2015); doi: 10.1063/1.4916509

View online: http://dx.doi.org/10.1063/1.4916509

View Table of Contents: http://scitation.aip.org/content/aip/journal/apl/106/12?ver=pdfcov

Published by the AIP Publishing

\section{Articles you may be interested in}

Sound absorption of a rib-stiffened plate covered by anechoic coatings

J. Acoust. Soc. Am. 137, 1551 (2015); 10.1121/1.4913782

Underwater sound scattering and absorption by a coated infinite plate with a distributed inhomogeneity

J. Acoust. Soc. Am. 133, 2082 (2013); 10.1121/1.4792353

Broadband sound absorption by lattices of microperforated cylindrical shells

Appl. Phys. Lett. 101, 184101 (2012); 10.1063/1.4764560

Analysis of absorption performances of anechoic layers with steel plate backing

J. Acoust. Soc. Am. 132, 69 (2012); 10.1121/1.4728198

Sound absorption and transmission through flexible micro-perforated panels backed by an air layer and a thin plate

J. Acoust. Soc. Am. 131, 3853 (2012); 10.1121/1.3701987

Model PS-100

Tabletop Cryogenic

Probe Station

\section{Lake Shore} CFYOTRONIOS

An affordable solution for a wide range of research 


\title{
Anomalous sound absorption in lattices of cylindrical perforated shells
}

\author{
Victor M. García-Chocano and José Sánchez-Dehesa ${ }^{\text {a) }}$ \\ Wave Phenomena Group, Department of Electronic Engineering, Universitat Politècnica de València, \\ Camino de Vera s.n. (Edificio 7F), ES-46022 Valencia, Spain
}

(Received 11 February 2015; accepted 17 March 2015; published online 26 March 2015)

\begin{abstract}
This work reports the enhancement of sound absorption by sonic crystals slabs made of cylindrical perforated shells. These building units, with perforations of millimeter size, show small losses and cannot explain the strong absorption observed at some specific frequencies when the slabs consist of just a few number of rows. It is found that this phenomenon is due to a resonant Wood anomaly which occurs when the incident wave couples with a leaky guided mode supported by the slab. This effect results in an enhancement of the absorption, since the energy transferred to the guided mode travels within the slab, along a direction perpendicular to the incident one. Multiple scattering and finite element simulations give support to the proposed behavior, the transmittance results being in good agreement with experimental data previously reported. (C) 2015 AIP Publishing LLC. [http://dx.doi.org/10.1063/1.4916509]
\end{abstract}

A lattice of solid rods in air is an example of a two-dimensional (2D) sonic crystal (SC) that has been extensively studied since the nineties. ${ }^{1-4}$ Most of the reported SCs were made of building units with negligible dissipation losses. However, in the last years, 2D SCs consisting of cylinders fabricated with lossy materials are deserving attention due to their potential application as acoustic barrier for broadband noise. ${ }^{5-10}$

The attention is paid here to $2 \mathrm{D}$ lattices of cylindrical perforated shells at normal incidence. These scattering units are obtained by rolling up perforated plates whose parameters (thickness, radius of the perforations, and perforation ratio) will determine the absorptive properties of the SC structure based on them. Thus, when the radius of the perforations is extremely small, viscosity effects inside the micro-pores lead to broadband attenuation spectra ${ }^{6}$ as occurs for the case of flat panels. ${ }^{11}$ However, if the perforation radius is of the order of hundreds of microns or more, the flat panels become acoustically transparent even for relatively low perforation ratios. The last effect was applied to obtain acoustically transparent cylindrical shells which were used as containers of granular absorbers. ${ }^{5}$ Though lattices of microperforated cylindrical shells have been comprehensively studied due to their application as broadband noise absorbers, ${ }^{6}$ no attention has been paid for the case of cylindrical shells whose perforation radii are too high to produce significant losses.

This letter studies the sound propagation properties through SC slabs made of square arrangements of perforated shells with small but non-negligible losses. These structures are characterized by a high transmission coefficient, while reflectance and absorption remain much smaller. However, at wavelengths approaching the lattice constant, a high reduction in the transmittance accompanied with an anomalous increase of the absorption is observed. It is shown here that the excitation of guided modes along the slab due to a Wood anomaly is the physical phenomenon behind the

\footnotetext{
${ }^{\text {a) }}$ Author to whom correspondence should be addressed. Electronic mail: jsdehesa@upv.es
}

observed behavior. Such a phenomenon was first observed by Wood in optical gratings ${ }^{12}$ and was subsequently explained by Rayleigh ${ }^{13}$ and Fano. ${ }^{14}$ Particularly, Fano identified two kinds of anomalies: (i) sharp or Rayleigh anomalies which occur when a new order of diffraction becomes propagative and (ii) resonant anomalies resulting from the interaction between the incident waves and leaky guided modes along the periodic surface. Wood's anomalies have been widely investigated for many years, a recent study of arrays of dielectric scatterers being an example closely related to the work here presented. ${ }^{15}$

The phenomenon here described is analyzed in two steps. First, the transmission properties of SC slabs are calculated by neglecting dissipation. Afterwards, the viscous losses of air are included, thus showing how dissipation plays a paramount role in order to explain the observed absorption enhancement, which is a direct consequence of the guided modes excited in the slabs.

The multiple scattering (MS) formalism is employed to calculate the transmission properties of lattices of perforated shells. In this framework, the scattering units are characterized by their $T$-matrix. The matrix elements $T_{q}$ are derived by applying the appropriate boundary conditions between the shells and the surrounding fluid, air in this case. Considering a perforated shell with external and internal radii $R^{+}$and $R^{-}$, respectively, the expression of its $T$-matrix is ${ }^{6}$

$$
T_{q}=-\frac{\rho_{q} J_{q}^{\prime}\left(k_{0} R^{+}\right)-J_{q}\left(k_{0} R^{+}\right)}{\rho_{q} H_{q}^{\prime}\left(k_{0} R^{+}\right)-H_{q}\left(k_{0} R^{+}\right)},
$$

where $k_{0}$ is the wavenumber in air and $\rho_{q}$ is

$$
\rho_{q}=\frac{J_{q}\left(k_{0} R^{-}\right)}{J_{q}^{\prime}\left(k_{0} R^{-}\right)}-\frac{i Z_{p} k_{0}}{\omega \rho_{0}}
$$

$\omega$ being the angular frequency and $\rho_{0}$ being the mass density of air. In these expressions, $J_{q}\left(H_{q}\right)$ and $J_{q}^{\prime}\left(H_{q}^{\prime}\right)$ denotes the Bessel (Hankel) function of $q$-th order and its derivative, respectively. $Z_{p}$ is the impedance of the flat perforated plate, which for the case of cylindrical holes is ${ }^{11,16,17}$ 


$$
\begin{aligned}
Z_{p}= & \frac{i \omega \rho_{0} t}{\sigma}\left[1-\frac{2}{s \sqrt{-i}} \frac{J_{1}(s \sqrt{-i})}{J_{0}(s \sqrt{-i})}\right]^{-1}+\frac{4}{\sigma} \sqrt{2 \eta_{0} \omega \rho_{0}} \\
& +\frac{i \omega \rho_{0}}{\sigma} \frac{16 r}{3 \pi}\left(1-2.5 \sqrt{\frac{\sigma}{\pi}}\right)
\end{aligned}
$$

where $s=r \sqrt{\left.\omega \rho_{0} / \eta_{0}\right)}$ is the perforate constant, $\eta_{0}$ is the dynamic viscosity of air, $t$ is the thickness of the plate, $r$ is the radius of the perforations, and $\sigma$ is the perforation ratio. The accuracy of this expression is supported by the experimental characterization of the perforated flat panels employed in the construction of the cylindrical shells. ${ }^{18}$

Let us consider a SC slab made of perforated shells with $R^{+}=4 \mathrm{~cm}, t=R^{+}-R^{-}=0.5 \mathrm{~mm}, r=0.25 \mathrm{~mm}, \sigma=14.5 \%$ arranged in a square distribution with lattice constant $a=11 \mathrm{~cm}$. These parameters correspond to those employed in Ref. 5 and will be used from now on. For an impinging plane wave with wavevector $k_{0}$ and a given angle of incidence, the reflectance $\mathcal{R}$ and transmittance $\mathcal{T}$ of a SC slab can be easily obtained using the formulas described elsewhere. ${ }^{3,6}$ The resulting total absorption $\mathcal{A}$ is then obtained by assuming energy conservation, i.e., $A=1-\mathcal{R}-\mathcal{T}$.

Figure 1(a) shows the transmittance of SC slabs with 1, 3 , and 5 rows, respectively, calculated for a normal-incident plane wave and taking the limit of vanishing viscosity $\left(\eta_{0}=0\right)$. It is observed that the transmittance is unity practically in the whole spectrum except around $3 \mathrm{kHz}$, where a minimum with zero-transmission appears. A zoomed view around this frequency is depicted in Fig. 1(b), which shows that total reflection is obtained even for the slab made of just one row of perforated shells. This behavior occurs at frequencies close to the diffraction limit $(\lambda=a)$, which is $3118.2 \mathrm{~Hz}$. The good agreement between the results from MS simulations (continuous lines) and those obtained using a 3D finite-element algorithm ${ }^{18}$ (symbols) supports the
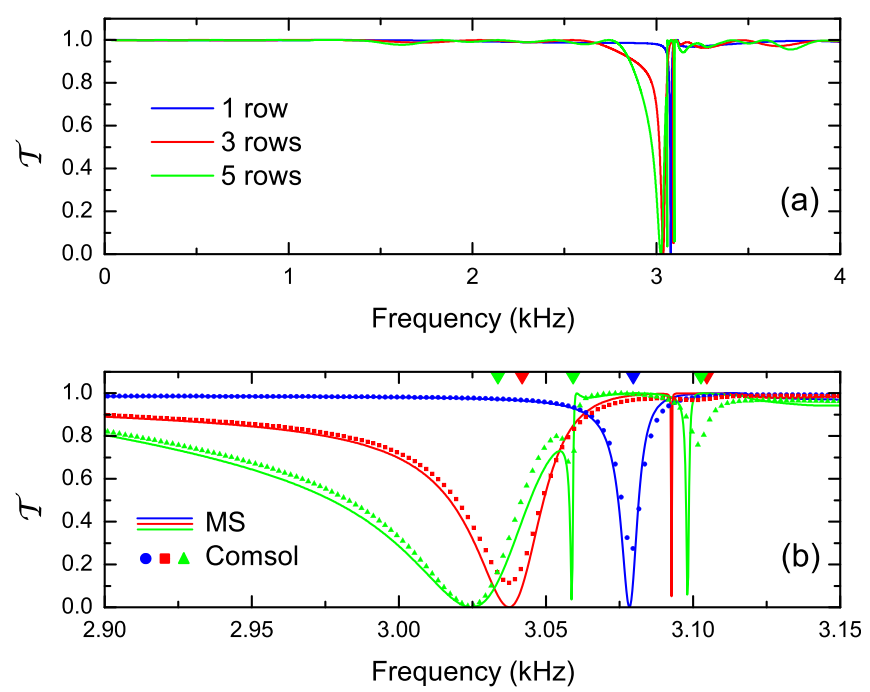

FIG. 1. Transmittance of lossless slabs with 1, 3, and 5 rows of perforated shells calculated through the multiple scattering theory (a) and zoomed view in the range $2.9-3.15 \mathrm{kHz}$ (b). The symbols correspond to numerical data obtained through 3D finite element simulations. The symbols in the upper part of figure (b) indicate the eigenfrequencies of the resonant states of the slabs. Note that the color of these symbols corresponds to the case having the same line color. validity of the analytical model introduced for the $T$-matrix (see Eq. (1)).

Figure 1(b) shows that the three transmittance spectra show a deep with an asymmetric profile typical from a Fano resonance. ${ }^{14}$ It evidences an interference phenomenon occurring between the transmitted sound waves and a resonant mode excited in the given slab. This effect points out the presence of a resonant Wood anomaly, the resonance corresponding to a leaky guided mode propagating along the slab, and therefore normally to the impinging direction. The position of this resonance is located at wavelengths slightly higher than the diffraction limit, as predicted for this type of anomalies. ${ }^{14}$ In addition, the position of the resonances shifts to lower frequencies for larger number of rows, proving the dependence between the resonant guided modes and the width of the slab. Besides the Fano resonance, additional sharp deeps can be observed for the slabs with 3 and 5 rows and are analyzed below.

The resonant modes of the slabs have been obtained through an eigenfrequency analysis of the 3D structure modelled in COMSOL. ${ }^{18}$ The frequency positions where resonances occur are indicated with arrows in the upper part of Fig. 1(b). The agreement found between the frequencies at which the transmission deeps appear and the calculated eigenfrequencies supports the explanation of the observed phenomenon through resonant Wood anomalies. Figures 2(a), 2(b), and 2(d) show the pressure field of the eigenmodes found at the first peak of transmittance in slabs with 1, 3,
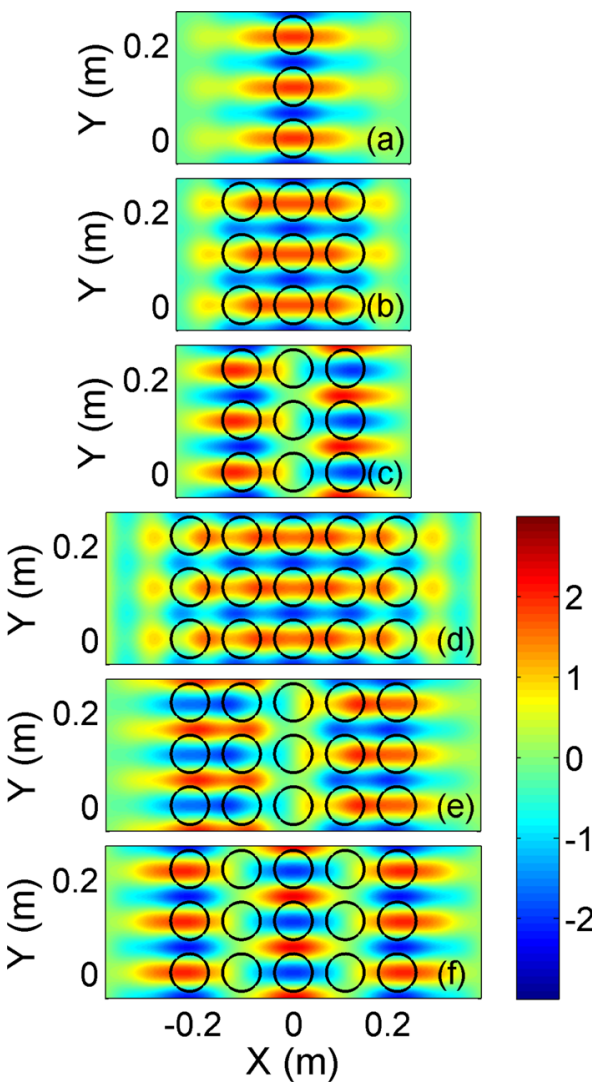

FIG. 2. The pressure field of the eigenmodes obtained through 3D finite element simulations for slabs with 1,3 , and 5 rows of perforated shells with no losses. The eigenfrequencies from figures (a)-(f) are $3079.7+3.9 i, 3041.9$ $+13.7 i, 3104.7+4.6 i, 3033.7+20.6 i, 3059.2+0.9 i$, and $3102.6+2.4 i$, respectively. 
and 5 rows of scatterers. A guided mode traveling through the interior of the slab is observed. It consists of a stationary wave based on the combination of two identical modes traveling along the $y$-axis in opposite directions. However, propagating modes that travel in a single direction can be observed in slabs excited with an incident sound whose wavefront has a finite cross-section. ${ }^{18}$

The extremely narrow deeps in Fig. 1(b) are associated with the eigenmodes illustrated in Figs. 2(c), 2(e), and 2(f). These pressure maps indicate that they correspond to resonances of higher-order. While the phase of the modes in Figs. 2(a), 2(b), and 2(d) keeps almost constant along the $x$-axis; in this case, two and three lobes appear within the slab. Similar conclusions can be drawn from MS calculations of slabs excited with a plane wave at similar frequencies. ${ }^{18}$ Note that the eigenfrequencies values are complex and are given in the caption of Fig. 2, their imaginary parts being related to the lifetime of the resonant modes. The lifetime of the low-frequency resonances decreases significantly with increasing number of rows. A low lifetime in slabs with many rows involves higher leaks from the guided mode to the free space, leading to wider resonances. The higher-order resonances have long lifetimes, which is consistent with the sharp profiles of the corresponding transmission deeps.

Now, let us study the case of SC slabs embedded in air with dynamic viscosity $\eta_{0}=17.8 \mu \mathrm{Pa} \cdot \mathrm{s}$. Figure 3 shows the reflectance $\mathcal{R}$, transmittance $\mathcal{T}$, and absorption $\mathcal{A}$ of slabs with 1,3 , and 5 rows obtained through multiple scattering calculations. Now the transmittance is not unity but its value is still high. The transmittance spectra present a single deep that appears at frequencies close to the diffraction limit and shifts to lower frequencies with increasing number of rows.

Therefore, the Wood anomalies still exists in lattices of perforated shells with viscous losses, although their amplitudes are smaller and their frequency response broadens due to the dissipation. It is also noticeable that the reflectance spectrum takes low values compared to absorption, even in the resonance where full reflection was found in the non-
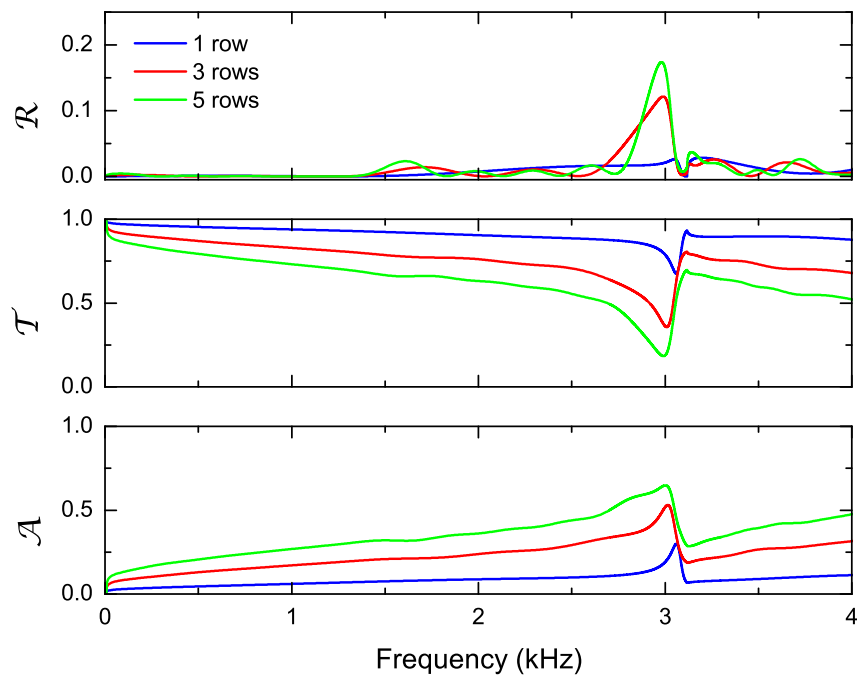

FIG. 3. Calculated reflectance $(\mathcal{R})$, transmittance $(\mathcal{T})$, and absorptance $(\mathcal{A})$ of SCs slabs with 1,3 , and 5 rows of perforated shells. The air viscosity is now taken into account. Notice that the reflectance is plotted in a narrow range for better visualization. lossy case. In this region, reflectance is enhanced, although the total attenuation of the slabs is mainly due to energy dissipation. As observed in the lossless case, the resonance anomaly involves a guided wave that travels along the axis of the slab. It is worth noting that the energy which is coupled to such guided mode travels perpendicular to the incidence direction along the interior of the slab. Thus, the sound is deviated from the free space to a path with low losses but which is infinitely long, in such a manner that it is slowly dissipated as it propagates. This results in the absorption enhancement observed in Fig. 3.

It is important to remark that the transmission spectrum shown in Fig. 3 matches with that experimentally observed in Fig. 7(b) from Ref. 5, which can be considered as an experimental proof of the phenomenon here reported. ${ }^{18}$

The coupling between the incident wave and the guided modes can be improved by increasing the filling fraction of the SC, i.e., by reducing the separation between cylinders. A sweep of the lattice parameter has been performed while the radius of the cylinders has been kept constant at $4 \mathrm{~cm}$. Figure 4 shows the corresponding 2D map of absorption calculated for a slab with three rows of scatterers as a function of the frequency and the filling fraction (ff) of the SC. Remember that $f f=\pi(R / a)^{2}$ for a square lattice. It is clearly observed the band where the anomaly occurs. It is located at slightly lower frequencies than the diffraction limit, which is indicated with a dash line. In addition to the first anomaly, a second branch can be observed. It corresponds to the frequency where the wavelength approaches one-half lattice parameter. The maximum absorption is found for the maximum filling fraction considered in the calculations $(f f=69.6 \%$ ), for which the separation between cylinders is $a=8.5 \mathrm{~cm}$. At this point, the absorption is $\mathcal{A}=0.77$ and the transmittance drops to $\mathcal{T}=0.08$. A similar behavior is found for the cases of 1 and 5 rows, whose minimum transmittance takes the values $\mathcal{T}=0.44$ and $\mathcal{T}=0.012$, respectively.

It is worth noting that an arrangement of two parallel plates has similar levels of absorption when compared to an arrangement of 1 row of perforated cylinders. Thus, the two parallel perforated plates would represent the front and rear

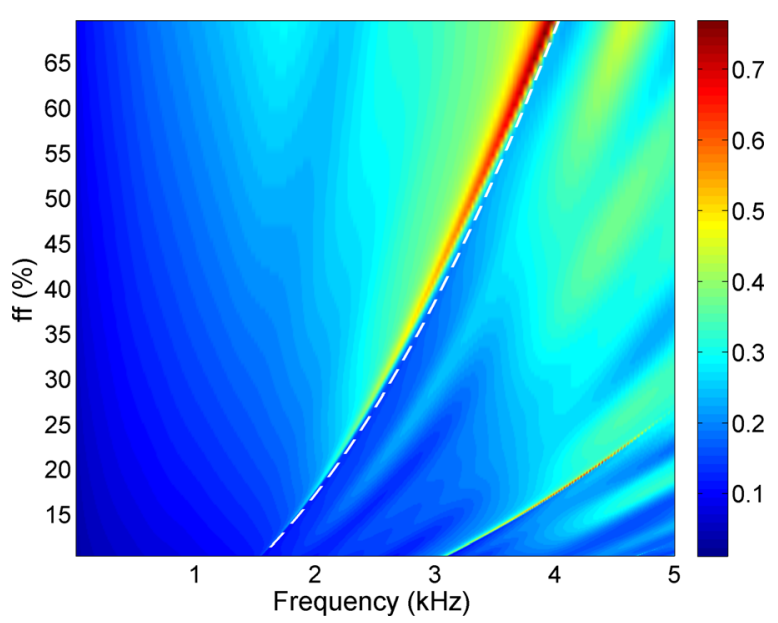

FIG. 4. Sound absorption of a slab of three rows of perforated shells as a function of the frequency and the filling fraction of the lattice. The cylinders are the same as those used in the previous figures, varying only the lattice parameter from $8.5 \mathrm{~cm}$ to $22 \mathrm{~cm}$ for the sweep of filling fraction. 

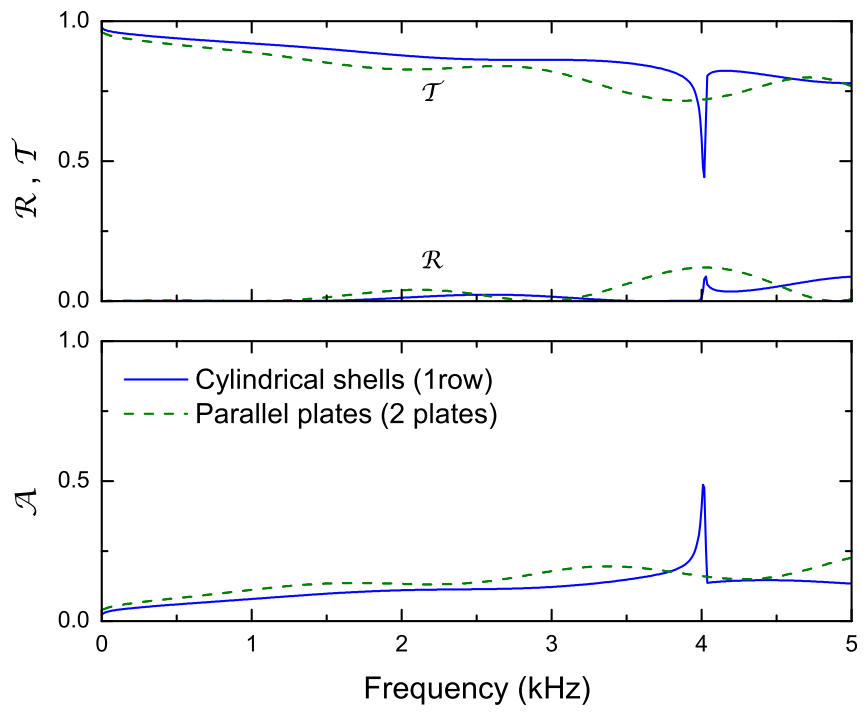

FIG. 5. Reflectance, transmittance, and absorption spectra of one row of perforated shells (solid lines) and an arrangement of two parallel perforated plates (dashed lines). The distance between nearest-neighbors cylinders and between the parallel plates is $8.5 \mathrm{~cm}$. Air viscosity has been considered in the calculations.

surfaces of the row of cylinders. As a 1D slab, it will show attenuation bands (or bandgaps) that depend on the separation between plates. However, the response of such a system is less selective. The implementation with cylinders results in attenuation mechanisms that are focused on a narrow band, allowing a high transmittance of sound at the remaining spectrum. An example is given in Fig. 5, where the reflectance, transmittance, and absorption of one row of shells are compared with those of two parallel perforated plates with similar type of perforations. It is observed that the overall transmittance of the slab of perforated shells is higher, although it presents a narrow peak where losses are highlighted.

In conclusion, this work has demonstrated that the sound attenuation observed in low lossy media consisting of lattices of perforated cylinders can be enhanced at specific frequencies due to guided modes excited near the diffraction limit. The resulting absorption is due to the strong dissipation experienced by the waves that are redirected along a direction perpendicular to the incident one. This type of Wood anomaly has potential application such as notch filters, where only a sharp specific band is intended to suppress.

This work was partially supported by the USA Office of Naval Research under Grant No. N000141210216 and the Spanish MINECO (Contract No. TEC2010-19751).

${ }^{1}$ J. V. Sánchez-Perez, D. Caballero, R. Martinez-Sala, C. Rubio, J. Sánchez-Dehesa, F. Meseguer, J. Llinares, and F. Gálvez, Phys. Rev. Lett. 80, 5325 (1998).

${ }^{2}$ C. Rubio, D. Caballero, J. V. Sanchez-Perez, R. Martinez-Sala, J. Sanchez-Dehesa, F. Meseguer, and F. Cervera, J. Lightwave Technol. 17, 2202 (1999).

${ }^{3}$ L. Sanchis, A. Hakansson, F. Cervera, and J. Sanchez-Dehesa, Phys. Rev. B 67, 035422 (2003).

${ }^{4}$ D. Torrent, A. Hakansson, F. Cervera, and J. Sánchez-Dehesa, Phys. Rev. Lett. 96, 204302 (2006).

${ }^{5}$ J. Sánchez-Dehesa, V. M. García-Chocano, D. Torrent, F. Cervera, S. Cabrera, and F. Simón, J. Acoust. Soc. Am. 129, 1173 (2011).

${ }^{6}$ V. M. García-Chocano, S. Cabrera, and J. Sánchez-Dehesa, Appl. Phys. Lett. 101, 184101 (2012).

${ }^{7}$ O. Umnova, K. Attenborough, and C. M. Linton, J. Acoust. Soc. Am. 119, 278 (2006).

${ }^{8}$ J. Christensen, V. Romero-García, R. Picó, A. Cebrecos, F. J. G. de Abajo, N. A. Mortensen, M. Willatzen, and V. J. Sánchez-Morcillo, Sci. Rep. 4, 4674 (2014).

${ }^{9}$ B. van der Aa and J. Forssén, J. Acoust. Soc. Am. 136, 2370-2380 (2014).

${ }^{10}$ V. Romero-García, J. V. Sánchez-Pérez, and L. M. García-Raffi, J. Appl. Phys. 110, 014904 (2011).

${ }^{11}$ D. Y. Maa, J. Acoust. Soc. Am. 104, 2861 (1998).

${ }^{12}$ R. W. Wood, Philos. Mag. 4, 396 (1902).

${ }^{13}$ J. W. S. L. Rayleigh, Proc. R. Soc. London 79, 399 (1907).

${ }^{14}$ U. Fano, J. Opt. Soc. Am. 31, 213 (1941).

${ }^{15}$ A. Maurel, S. Félix, J. F. Mercier, A. Ourir, and Z. E. Djeffal, J. Eur. Opt. Soc. Rap. Public 9, 14001 (2014).

${ }^{16}$ U. Ingard, J. Acoust. Soc. Am. 25, 1037 (1953).

${ }^{17}$ S. Allam and M. Åbom, J. Vib. Acoust. 133, 031005 (2011).

${ }^{18}$ See supplementary material at http://dx.doi.org/10.1063/1.4916509 for details of the numerical simulations. 